\title{
Research on the Sunken Resources Allocation of Coal Mine Production Logistics
}

\section{System Based on Inverse Optimization}

\author{
Liu Peng, Wang Jinfeng \\ Zhengzhou University, Henan Zhengzhou, China \\ 2282968615@qq.com
}

Keywords: coal mine production logistics system; sunken resource allocation; inverse optimization; safety

\begin{abstract}
Considering the irreversibility of the safety resources in the coal mine production logistics system, this paper firstly proposes the concept of the safety resource of the coal mine production logistics system. Then this paper constructs the optimal allocation model of the sunken safety resource of the coal mine production logistics system, which is used to allocate the budget of the sunken safety resources in the early stage. Based on this, this paper constructs the inverse optimization model of the safety resource of the coal mine production logistics system, which realizes the promotion of the safety level of coal mine production logistics system again under the situation that the sunken resources cannot be reallocated. Finally, the feasibility of the model is verified through an example. The result shows that the inverse optimization model of the sunken safety resource of the coal mine production logistics system can improve the safety level under the condition that the input of the safety resource is irreversible, which has important practical significance for the coal mine safety production work.
\end{abstract}

\section{INTRODUCTION}

Coal mine is the lifeblood of our national economy, and the widespread use of coal mine promotes the construction process of our national economy, improves people's living standards and will continually make the significant contribution. However, the gap of coal mine security production level between China and developed countries is huge. For example, the death rate of Chinese coal industry was 0.293 per million tons which is 10 times that of advanced countries in 2013. The high mortality rate and high accidents frequency in the field of coal mine industry has been a problem in coal mine production in China all the time.

Compared with other industries, coal mine production has its obvious characters. Coal mine production process includes physical form changes of the coal (mining and crushing) spatial position change of coal mine (transport under the shaft and on the ground), which actually is a special logistics process (Jinfeng Wang, Xueqi Zhai,2014). In addition, there are many kinds of material and substance flow like equipment, material, wind flow in the process of coal mine production. And these constitute the coal mine production logistics system which is the main part of coal production. As the important part of coal mine production, coal mine production logistics plays a huge impact on security production in coal mine. Therefore, in order to improve the safety level of coal mine production and eliminate the hidden trouble of safety, it is necessary to optimize the allocation of safety resources in coal mine production.

At present, there are many achievements in the research on the safety resource allocation of the coal mine production logistics system. (He Gang, with others,2008) used system dynamics theory to optimize the security investment direction for government and coal mine enterprise to reduce the accident rate due to people and improves the security level of coal mine production. According to features in coal mine security investment, (Ai-hua Zheng, and others,2005) elaborate the economic issues such as the shortage of input power of coal mine security, diseconomy of security investment and so on, and propose appropriate measures based on this. (Jinfeng Wang and others,2015) use stochastic programming and cooperative game theory to construct Security resource allocation optimization model of coal mine production logistics system, and achieve the optimal allocation of security resources. The above optimal methods play a good effect on enhancing the security level of coal mine production logistics system. However, because of the irreversible property of security resources in coal mine production logistics system, it is difficult to reallocate security resources once they are input into the system, and few studies take into account this feature.

Coal mine security resources cannot be reallocated once they begin to play a role. The essence of optimizing the security resources can be classified as inverse optimization problem. Under a set of definite solutions, the inverse optimization can make the target value reach the ideal target value by adjusting the operating parameters of the original optimization model. The inverse optimization method has been applied to many fields and achieved good results. On the purposes of optimizing information sharing process on the basis of not disclose personal information, (P. Richard,2011) constructs inverse optimal model of joint economic lot for the overall supply chain performance problems. (V. T. Alexander,2011) takes motor control redundancy problem as the research object, and uses linear and inverse optimization method to achieve the purpose of the cost optimization in motor control. (Xiangbin Zhang, 2011) has developed a support system of supply chain resource optimization allocation based on linear programming inverse optimization model, and realizes 
the rapid optimization of supply chain resources facing the market. (Jin Chun,2013)

completes the inverse optimization by adjusting resource efficiency matrix, which translates the original feasible solution of established resource allocation model into the optimal solution.

Above all, in the existing research results, the inverse optimization methods, which are feasible to optimize the allocation of resources, optimize the original model to the target value by adjusting the model parameters, and solve the problem of resource allocation optimization in many fields. However, most of these models are linear programming models, and the strong nonlinear relationship between the security resources and the security state should be taken into account in the allocation of resources. Therefore, this paper intends to establish the nonlinear inverse optimization model of coal mine production logistics system to solve the problem of optimizing the allocation of coal mine production logistics system.

\section{RELATED THEORY}

Analysis of coal mine production logistics system resources

Sunken resources are those resources that have occurred in the past, and cannot be changed by any decision now or in the future. In the process of reproduction, there will always be a part of resources, such as time, money, energy and so on. After the investment in the early stages of the production, the allocation of these resources will not be able to be recovered or changed the way of allocation once the production starts (Yanying Chen,2012).

According to the relevant research and system engineering theory, the factors that affect the security of coal production logistics system could be divided into five parts which include equipment factor, operating factor, management factor, human capital factor and environmental factor (Jinfeng Wang,2014). The equipment factor refers to the coal mine production, storage and transportation machinery and equipment level. Operating factor refers to operation process of the production operators and mining schemes made by the management organization. Management factor refers to the design of the organizational structure of the coal production enterprises, the rules and regulations formulation and the characteristics of enterprise culture. Human capital factor refers to the ability and quality of production personnel. Environmental factor includes geology, meteorology and other environmental characteristics of mineral deposits. The logistics system of coal mine production inputs the security resource according to the above factors, as shown in Figure 1.

Most of the safety investment in the production logistics system of coal mine is the sunken resources. The coal mine enterprise usually carries on the budget to the safety input before the production work, and makes the safety output to achieve the maximum through the security resources optimization allocation. When production begins, security resources begin to play a role. Because the coal mine safety resources are mainly used to buy and maintain the facilities and equipment, maintenance, the safety resources can be sustained for a long time after the investment, and once the safety resources are put into production they cannot be recovered. Therefore, the resource input of coal mine production logistics system has strong irreversibility, which belongs to the category of the sunken resources.

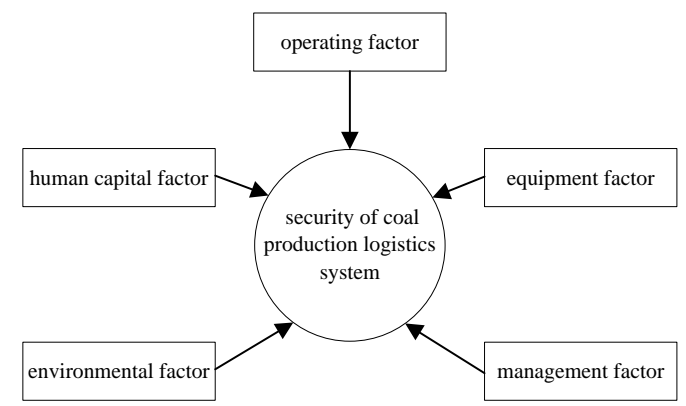

Fig. 1 Security influencing factors of coal mine production logistics system resources

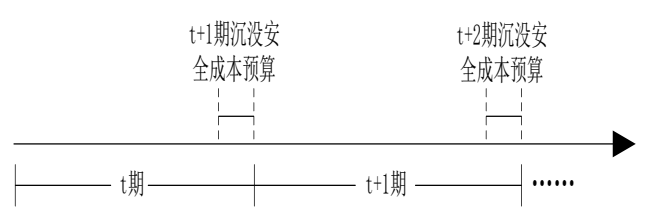

Fig. 2 Flow chart of the sinking security resources

As shown in Figure 2, During the period of the safety resources in $t+1$ period are allocated at the end of the $t$ period, which are worked in the $t+1$ period, and the sunk safety resource input could not be redistributed in the $\mathrm{t}+1$ period.

\section{CONSTRUCTION OF THE INVERSE OPTIMIZATION MODEL OF THE SUNKEN RESOURCES OF COAL MINE PRODUCTION LOGISTICS SYSTEM}

\subsection{Model assumptions}

Before building the model, make the following assumptions:

Assuming 1: The importance of the security output could be changed with the work adjustment of the security resources input later period;

Assuming 2: All the security resources of the coal mine production logistics system cannot be changed after the function

Assuming 3: The total amount of security resources input is limited.

\subsection{Model construction}

\subsubsection{Construction of the forward optimization model}

In order to maximize the efficiency of the sunk resources, the coal enterprises can allocate the sunk resources by constructing the forward optimization model before the safety sunk resources are invested.

First of all, according to the results of literature researches, Cobb-Douglas production function could better reflect the relationship between security input and 
security output. Therefore, this paper takes security maximum output as objective function, and constructs the objective function according to the types of coal mine sunk resources. The objective function is:

$$
\operatorname{Max} Y=A x_{1}^{\alpha_{1}} x_{2}^{\alpha_{2}} x_{3}^{\alpha_{3}} x_{4}^{\alpha_{4}} x_{5}^{\alpha_{5}}
$$

In the function, $A$ is constant, $x_{1}$ is the security input level of equipment factor, $x_{2}$ is the security input level of operation factor, $x_{3}$ is the security input level of management factor, $x_{4}$ is the security input level of human capital factor, $x_{5}$ is the security input level of environmental factor. $\alpha_{1}$ is the important degree of safety input for equipment factor, $\alpha_{2}$ is the importance degree of safety input for operation factor, $\alpha_{3}$ is the important degree of safety input for management factor. $\alpha_{4}$ is the important degree of safety input for human capital factor. $\alpha_{5}$ is the important degree of safety input for environmental factor.

Secondly, there will be some constraints in the process of the security sunken resource allocation, and the resources must be allocated in the scope of these constraints to meet the requirement of the normal production:

(1) Equipment and environmental factors represent the facilities investment of security production. Too little hardware security input will make the security be difficult to be guaranteed so equipment and environmental factors input should not be less than $B_{1}$, which can be shown as follows:

$$
x_{1}+x_{5} \geq B_{1}
$$

(2) As the input related to people closely in security production, operation, management, and human capital factors also need a certain amount of capital investment as the guarantee, so the input of operational factor, management factor, human capital factor should not be less than $B_{2}$, which could be shown as follows:

$$
x_{2}+x_{3}+x_{4} \geq B_{2}
$$

(3) Coal production enterprises make the investment budget of production safety in the early stages, and make expenditure of safety investment according to the budget. Therefore, the input of equipment factor, operation factor, management factor, human capital factor and environmental factor should not exceed the total budget, which could be shown as follows:

$$
x_{1}+x_{2}+x_{3}+x_{4}+x_{5} \leq B_{3}
$$

(4) In order to ensure that each factor could be carried out normally, the input of each factor has a lower $\operatorname{limit}\left(C_{i}\right)$ :

$$
x_{i} \leq C_{i}
$$

At last, according to the above objective function and constraint conditions, the forward optimal allocation model of coal mine production enterprises of the security sunken resources investment in the early stage is obtained:

$$
\begin{aligned}
& \operatorname{Max} Y=A x_{1}^{\alpha_{1}} x_{2}^{\alpha_{2}} x_{3}^{\alpha_{3}} x_{4}^{\alpha_{4}} x_{5}^{\alpha_{5}} \\
& \text { s.t. }\left\{\begin{array}{l}
x_{1}+x_{5} \geq B_{1} \\
x_{2}+x_{3}+x_{4} \geq B_{2} \\
x_{1}+x_{2}+x_{3}+x_{4}+x_{5} \leq B_{3} \\
x_{i} \leq C_{i}
\end{array}\right.
\end{aligned}
$$

According to the formula (6), the enterprises optimize the security sunken resources to achieve optimal effect of the security sunken resources input.

\subsubsection{Construction of Inverse Optimization Model}

Solving the forward security sunken resources allocation optimization model constructed by the enterprise through the formula (6) can get a set $\left(X^{*}\right)$ that make the security output to be maximum ( $\operatorname{Max} Y$ ). At the same time, enterprises input security sunken resources according to $X$. However, after the beginning of the production cycle, security output ( $\operatorname{Max} Y$ ) may not meet the current security demand, that's Max $Y \leq Z^{*}$. Then the enterprise needs to use the inverse optimization method to solve this problem (Jinfeng Wang,2015).

Inverse optimization can make the original objective function tend to a certain value by changing the model parameters on the basis of an original solution. The model formula (6) could be transformed into an inverse optimization model (7):

$$
\begin{aligned}
& \min Y=\left(A x_{1}^{* \alpha_{1}} x_{2}^{* \alpha_{2}} x_{3}^{* \alpha_{3}} x_{4}^{* \alpha_{4}} x_{5}^{* \alpha_{5}}-Z^{*}\right)^{2} \\
& \text { s.t. }\left\{\begin{array}{l}
\alpha_{1}+\alpha_{2}+\alpha_{3}+\alpha_{4}+\alpha_{5}=1 \\
c_{L i} \leq \alpha_{i} \leq c_{U i}
\end{array}\right.
\end{aligned}
$$

In the model, $C_{L i}$ is the lower limit of the importance degree of resource $i, C_{U i}$ is the upper limit of the importance degree of resource $i$.

Now, the formula (7) can be seen as the inverse optimization model of security sunken resources of coal mine production logistics system. In the case of a set of optimal feasible solution of the original model, this model can make security to achieve $Z^{*}$ through adjusting the importance degree of each factor to improve the safety of coal mine again.

\section{EMPIRICAL ANALYSIS}

This paper takes the mine $\mathrm{C}$ affiliated to a Coal Mine Group in Henan as the empirical object. The annual production capacity designed of the mine is 1 million tons and the service life is 20 years. This mine fields adopt inclined, vertical shaft mixed developing method to explore the coal, which include the main inclined shaft, auxiliary inclined shaft, return air inclined shaft. The ventilation mode of the mine is the central juxtaposition mode, and ventilation method uses mechanical extraction. This mine improves the overall security level due to the 
increase input on personnel security training and the introduction of advanced mining equipment. The security level of the mine continues to improve because of the successive increase on the security input.

In order to ensure the smooth progress of the security production, the mine plans to invest 3.27 million RMB for coal production logistics system in five aspects which include equipment factor, operation factor, management factor, human capital factor, environmental factor this year

According to previous data, and combined with the formula (6), this paper fits the relationship between the security output and the security input first, and then gets the inverse optimization model of security sunken resources of coal mine production logistics system of mine $\mathrm{C}$ :

$$
\begin{aligned}
& \operatorname{Max} Y=1.32 x_{1}^{0.213} x_{2}^{0.211} x_{3}^{0.152} x_{4}^{0.226} x_{5}^{0.198} \\
& \text { s.t. }\left\{\begin{array}{l}
x_{1}+x_{5} \geq 165 \\
x_{2}+x_{3}+x_{4} \geq 110 \\
x_{1}+x_{2}+x_{3}+x_{4}+x_{5} \leq 327 \\
x_{1} \geq 80 \\
x_{2} \geq 50 \\
x_{3} \geq 60 \\
x_{4} \geq 40 \\
x_{5} \geq 80
\end{array}\right.
\end{aligned}
$$

Due to the irreversibility of security resources input, mine $C$ uses the model (8) to make the budget on the input of the security resources before the start of this year's production. Use Matlab genetic algorithm to solve model. When $\mathrm{X}=[82.780,53.313,63.536,44.922$, $82.432]$, the security output is 84.625 . Therefore, this year's investment in security equipment factor, operation factor, management factor, human capital factor, environmental factor are respectively 82.78 million RMB 53.313 million RMB, 63.536 million RMB, 44.922 million RMB, 82.432 million RMB, and the maximum security output that the $C$ mine could achieve is 84.625 .

C mine starts this year's work according to the budget of the above security sunken resources allocation. However, with the continuous improvement of the security level requirement, security output needs to reach 87 to meet the requirement of the current security production in the work process. In this regard, the enterprise can construct the inverse optimization model of sunken resources according to the formula (7), and the model is as the follows:

$$
\begin{aligned}
& \min Y=\left(1.32 * 82.780^{\alpha_{1}} 53.313^{\alpha_{2}} 63.536^{\alpha_{3}} 44.922^{\alpha_{4}} 82.432^{\alpha_{5}}-87\right)^{2} \\
& \text { s.t. }\left\{\begin{array}{l}
\alpha_{1}+\alpha_{2}+\alpha_{3}+\alpha_{4}+\alpha_{5}=1 \\
0.15 \leq \alpha_{1} \leq 0.3 \\
0.15 \leq \alpha_{2} \leq 0.3 \\
0.15 \leq \alpha_{3} \leq 0.3 \\
0.15 \leq \alpha_{4} \leq 0.3 \\
0.15 \leq \alpha_{5} \leq 0.3
\end{array}\right.
\end{aligned}
$$

Use the genetic algorithm to solve the model. When $\alpha_{i}=[0.220,0.181,0.197,0.159,0.243]^{T}$, the safe output could reach 87 so as to meet the requirements of security production.

Table 1 Variety of the importance of each factor

\begin{tabular}{cccc}
\hline Factors & $\begin{array}{c}\text { Before the Inverse } \\
\text { Optimization }\end{array}$ & $\begin{array}{c}\text { After the Inverse } \\
\text { Optimization }\end{array}$ & Variety \\
\hline Equipment factor & 0.213 & 0.220 & +0.007 \\
Operation factor & 0.211 & 0.181 & -0.030 \\
Management Factor & 0.152 & 0.197 & +0.045 \\
Human Capital Factor & 0.226 & 0.159 & -0.067 \\
Environmental Factor & 0.198 & 0.243 & +0.045 \\
\hline
\end{tabular}

According to the inverse optimization results in Table 1 , it is necessary to improve recognition degree of the equipment factor, management factor and environmental factor in the production process and enhance the importance level of these factors in the process of safety production to realize the improvement of the security outputs.

\section{CONCLUSIONS}

For the irreversibility of security resources in coal mine production logistics system, this paper constructs the inverse optimization model of security sunken resources of coal mine production logistics system realize the improvement of the security level and verifies the validity of the model is verified through an example. The results show that inverse optimization model of security sunken resources of coal mine production logistics system will enhance the security level of the coal mine under the condition that security sunken resources had already been input, and it has the important practical significance to security work of coal mine production.

\section{ACKNOWLEDGEMENTS}

This study is supported by grants from Key Research Programs in Colleges and Universities of Henan Province (Nos.17A630059).

\section{REFERENCES}

[1] Aihua Zheng, Nie Rui, 2005.The perspective of coal mine safety investment strategy from the frequent mine disasters[J]. Economic Management.11:90-93. 
[2] He Gang, Guoshu Zhang, Qinghua Chen, Guotong Qiao, 2008.A system dynamics(SD) simulation analysis on the influencing factors of human behavior in coal mine work safety[J]. China Safety Science Journal.09:43-47.

[3] Jinfeng Wang, Xueqi Zhai, Lijie Feng,2014. Efficiency optimization of coal mine production logistics under safety hard constraint [J]. Chinese Journal of Management Science. 07:59-66.

[4] Jinfeng Wang, Xueke Du, Lijie Feng, Xueqi Zhai,2015. Safety resource allocation model of coal mine production logistics system using stochastic programming[J]. China Safety Science Journal.03:16-22.

[5] Jinfeng Wang, Xueke Du, Xueqi Zhai, Lijie Feng, 2015.Research of resource allocation of coal mine production logistics system based on cooperative game[J]. Industry and Mine Automation.12:25-30.

[6] Jin Chun, Tingting Hu, Weizhen Rao, Qiunan Meng,2013. Inverse optimization model of linear programming for operation resources configuration on logistics center[J]. Industrial Engineering and Management.03:56-61.

[7] Jinfeng Wang, Zhang Bo, Lijie Feng, Xueqi Zhai, 2014.System dynamics modeling and demonstration of safety level of production logistics of coal mine[J]. Industrial Engineering and Management.08:34-38.

[8] Jinfeng Wang, Yunfei An, Xueqi Zhai, Lijie Feng, 2015.Study on security resources allocation of coal mine production logistics system based on inverse optimization[J]. Industrial Engineering and Management.05: 137-142.

[9] P. Richard, Y. Y. Zhang, Florian Kerschbaum, Axel Schröpfer, 2011.Secure collaborative supply chain planning and inverse optimization-The JELS model[J]. European Journal of Operational Research. (208):75-85.

[10] V. T. Alexander, M. Z. Vladimir, 2011.Analytical and numerical analysis of inverse optimization problems: conditions of uniqueness and computational methods[J]. Biological Cybernetics. (104):75-93.

[11] Xiangbin Zhang, 2011.Research on supply chain resources optimal allocation support system based on linear programming inverse optimal models[J]. Journal of Nanjing University of Posts and Telecommunications (Natural Science).31(1):96-101. [12] Yanying Chen, Zongke Bao, 2012.Learning by doing and the market structure of Chinese manufacturing: from the perspective of endogenous sunk cost[J]. China Industrial Economics.08:43-55. 